\title{
Thanks to JMCP Peer Reviewers, 2018
}

The Journal of Managed Care \& Specialty Pharmacy would like to thank the 291 reviewers who have evaluated manuscripts in 2018 and contributed to the high quality of articles in JMCP. Without this excellent level of reviewer involvement, $J M C P$ would not be one of the most respected journals in health care services, connecting authors and innovators to accelerate advances in managed care pharmacy.

Susan Abughosh, PhD

Sarah Elaine Adkins, BS, RPh, PharmD

Medinat S. Akinbi, PharmD

James Daniel Allen, PharmD

Aylin E. Altan, PhD

JaeJin An, BPharm, PhD

Bithia Anderson, PharmD, MPA

Jennifer Andres, PharmD

Rebekah Hanson Anguiano, PharmD, BCPS

Chukwuma U. Anyanwu, PharmD, MPH, MBA

Sheta Ara, PharmD

Hilary A. Aroke, MD, MPH

Adenike Atanda, PharmD

Steve G. Avey, MS

Danial E. Baker, BPharm, PharmD

Jennifer Waymier Baker, PharmD, BCPS, BCACP

Jamie C. Barner, PhD

Mitchell J. Barnett, PharmD, MS

Ramsankar Basak, PhD

Christopher Bell, MS

Brandon K. Bellows, PharmD, MS

Todd Berner, MD

Gary Besinque, PharmD, FCSHP

Abhijeet Bhanegaonkar, PhD, MPH

Seema Bhatlekar, PhD, MBA

Sandipan Bhattacharjee, BPharm, MS, PhD

Sarah J. Billups, PharmD

Mary Ellen Bonk, PharmD

Matthew E. Borrego, PhD

Marc F. Botteman, MSc

Brian Bouvier, PharmD

Joel Brill, MD

Diana Brixner, PhD

Courtney Rhiannon Brown, PharmD
Dana N. Brown, BS, PharmD

Joshua David Brown, PharmD, PhD

Amanda Brummel, PharmD

Ami R. Buikema, MPH

Russel Burge, PhD

Eric Burton, MD

Stacey Dacosta Byfield, PhD, MPH

Nicole Caffiero, PharmD

H. Eric Cannon, PharmD

Patricia Carlson, PharmD

Javier Carrera, PharmD

Norman V. Carroll, PhD

Sara Carruth, PharmD

Swetha Challagulla, MS

Vivien Chan, PharmD

Kevin Chang, PharmD

Satabdi Chatterjee, MS, PhD

Shraddha Chaugule, PhD

Hua Chen, PhD, MD

Judy W. Cheng, PharmD

Donna Chiefari, BScPharm, PharmD

Chanadda Chinthammit, BSPharm, MS

Shelley Chun, PharmD

Robert Colditz, PharmD

Jordan R. Covvey, PharmD, PhD, BCPS

William Crown, PhD

Brian Keith Crownover, MD

Eric J. Culley, PharmD, MBA

Desola Davis, PharmD

Devender Dhanda, PhD, MBA, MS

Emmanuel Fulgence Drabo, PhD

Aaron Douglas Drovandi, MPharmPH

Dana Angela Drzayich Antol, MS

Joseph Ted Dye, PhD, RPh

Michael T. Eaddy, PharmD, PhD
Lisa A. Edwards, PharmD, RPh

Frank R. Ernst, PharmD, MS

Joel F. Farley, PhD

Beckie A. Fenrick, PharmD

Marc L. Fleming, PhD

Renee R. Fleming, RPh, MBA

Sara Forrester, PharmD, MS

Meg A. Franklin, PharmD, PhD

Sharon Glave Frazee, PhD, MPH

Jennifer Friderici, MS

Brian John Gates, PharmD

Justin Gatwood, PhD, MPH

Teresa B. Gibson, PhD

Jasjit Gill, BSPharm, PharmD

Peter Glassman, MBBS, MSc

Patrick P. Gleason, PharmD, BCPS

Chester Bernie Good, MD, MPH

Jennifer S. Graff, PharmD

Bonnie C. Greenwood, PharmD, BCPS

Scott Karr Griggs, PharmD, PhD

Paul 0. Gubbins, PharmD

Brooke Harrow, PhD

Amany K. Hassan, PhD

Noelle K. Hasson, PharmD

Joel W. Hay, PhD

Gregory Heindel, PharmD

Henry J. Henk, PhD

Brian Henriksen, $\mathrm{PhD}$

Inmaculada Hernandez, PharmD, PhD

Lisa E. Hines, PharmD

Connie Ho, PharmD, MBA

Mei Jen Ho, PharmD, MSPH

Natalie S. Hohmann, PharmD

Anke-Peggy Holtorf, PhD, MBA

James Hopsicker, RPh, MBA 


\section{Thanks to JMCP Peer Reviewers, 2018 (continued)}

Rita L. Hui, PharmD, MS

Anna Hung, PharmD, PhD, MS

Srihari Jaganathan, MS

Tara Kukuschkin Jellison, PharmD, MBA

Ruixuan Jiang, BS

Yawen Jiang, PhD

Tianze Jiao, PhD

Aditi Kadakia, MS

Pravin Shivaji Kamble, BPharm, MTech,

$\mathrm{RPh}, \mathrm{MS}, \mathrm{PhD}$

Hyeun Ah Kang, MS

Jasmine Kaur, PhD

Shellie L. Keast, PharmD, PhD

Tasleem Khansia, PharmD

Kibum Kim, PhD

Yoona Kim, PharmD, PhD

Donald G. Klepser, PhD, MBA

Stephen J. Kogut, MBA, PhD

Mark J. Kohn, PharmD, BCPS

Yu Heng Kwan, BScPharm

Pamela Landsman-Blumberg, DrPH

Kenneth LaPensee, PhD, MPH

Ken A. Lawson, PhD

Emily Leckman-Westin, PhD

Jeff Lee, PharmD, FCCP

Thomas Lee, PharmD

YoonJung Lee, PharmD

Melissa Leedock, PharmD

Premila Leiphrakpam, MBBS, PhD

Matthew David Lennertz, PharmD, MS, MBA

Kimberly Lenz, PharmD

Christine Leong, PharmD

Haitao Li, PharmD, MS

Nanxin Li, PhD, MBA

Joshua N. Liberman, PhD

Jay Lin, PhD, MBA

Carroline Priya Lobo, MS, PhD

F. Ellen Loh, BPharm, PhD, MBA

Gregory A. Low, BPharm, PhD

Junjie Ma, MS, PhD candidate

Gary P. MacDonald, D0, MS, FAAFP

Elizabeth A. MacLean, PharmD, PhD
Jonathan W. Magness, PharmD

Rohan Mahabaleshwarkar, PhD

Daniel C. Malone, PhD

Amanda Mann, PharmD

Bradley C. Martin, PharmD, PhD

Josephine A. Mauskopf, PhD

Carrie McAdam-Marx, PhD, RPh

Katharine McCarthy, PharmD

Jeffrey Scott McCombs, PhD

Cara McDermott, PharmD, PhD

Tracy McDowd, PharmD

Scott Thomas McDowell, PharmD, BCPS, CDE

Darshan Mehta, MS

Brian L. Meissner, PharmD, PhD

Andrew Merker, PharmD

Shivani Mhatre, PhD

Daryl E. Miller, PharmD

Lesley-Ann Miller-Wilson, MS, PhD

Manish Mittal, PhD

Fumihiro Mizokami, PhD

Uche Anadu Ndefo, PharmD

Daniel B. Ng, PharmD, MBA

Chi Nguyen, PhD

Weiyi Ni, PhD

Mary 0. Obeng, PharmD

Rachel Roberts Ogden, PharmD, MHSA

Richard L. Ogletree, Jr., PharmD

Arinze Nkemdirim Okere, PharmD, MS, MBA, BCPS

Abiola 0. Oladapo, $\mathrm{PhD}$

Busuyi Olotu, PhD

Oluwadamilola Onasanya, MD, MPH

Kelsey Ondek, PharmD

Gary Owens, MD

Nancy M. Pace, BS, PharmD, MBA

Neha Sheth Pandit, PharmD

Harish Singh Parihar, PhD

Haesuk Park, PhD

Sharon K. Park, PharmD

Si Yeon Park, PharmD

Yujin Park, PharmD
Anisha M. Patel, PhD, MS, BPharm

Vardhaman Patel, MS, PhD

Allison Petrilla, MPH

Tracy K. Pettinger, PharmD

Timothy Pham, PharmD, PhD

Pedro Plans-Rubio, MD, PhD, MSc

Thomas C. Pomfret, PharmD, MPH, BCPS

Gerald Pulver, PhD, MPH

Lauren Pusateri-Nilson, PharmD

Thomas Scott Raisor, PharmD, MPH, MBA

Rajendiran Rajesh, PhD

Jigar Ramesh Rajpura, PhD

Amit D. Raval, PhD, MPharm

Prabashni Reddy, PharmD

Claiborne (Gene) E. Reeder, RPh, PhD

Victor I. Reus, MD

Taeho Greg Rhee, PhD, MSW

Terry Richardson, PharmD, BCACP

M. Christopher Roebuck, MBA, PhD

Lisa Rotenstein, MD, MBA

Deanna M. Rowe, PharmD

Karine Rozenberg-Ben-Dror, PharmD

Elan Rubinstein, PharmD, MPH

Jim Ruble, PharmD, JD

Michael T. Rupp, PhD, BSPharm, FAPhA

Sheila Ryder, BScPharm, MScCommPharm, PGDipStat, PhD candidate

Prashant Sakharkar, PharmD, MPH

Vishal H. Saundankar, MS, BPharm

Kimberly Saverno, PhD, RPh

Phil Schwab, PhD

Julie M. Sease, PharmD

Jordan Sedlacek, PharmD

Todd Sega, PharmD

Mark S. Shaefer, PharmD

Ruchit Shah, PhD

Sanket Shah, MBBS

Qiujun Shao, BS

Blake Shay, PharmD, MS, BCPS

Natalia G. Shcherbakova, PhD

Xian Shen, PhD

Marvin D. Shepherd, PhD 


\section{JMCP}

\section{Thanks to JMCP Peer Reviewers, 2018 (continued)}

Katarzyna Shields, PharmD, MBA

Jonathan Silver, MD, MS, MBA

Jason C. Simeone, PhD

David Singer, PharmD

Mukul Singhal, PhD, BPH

Julia F. Slejko, PhD

David Michael Sloan, PhD

Amy Smalarz, PhD, MHA

Mary Small, MS

Jennifer G. Smith, PharmD

Judith A. Smith, PharmD, BCOP, CPHQ,

FCCP, FISOPP

Leah S. Smith, PharmD, BCPS, CGP

Margie E. Snyder, PharmD, MPH

Marilyn Stebbins, PharmD

David Stenehjem, PharmD

Craig Stephen Stern, RPh, PharmD, MBA

James G. Stevenson, PharmD, FASHP

Samuel Stolpe, PharmD, MPH

Bradley S. Stolshek, PharmD

Karyn Michelle Sullivan, PharmD, MPH

Kent Summers, PhD
Satya Surbhi, PhD, MS

Amy Suss, PharmD

Patty Taddei-Allen, PharmD, MBA

Jeffery Charles Talbert, PhD

Xi Tan, PhD, PharmD

Reginald Tayaben, PharmD

Sarah Thayer, PhD

Ashley Thomas, PharmD

Tara M. Thomas, RPh

Daniel Mark Tomaszewski, PharmD, PhD

Ivy Q. Tonnu-Mihara, PharmD, MS

Stephanie Tran, PharmD

Benjamin Y. Urick, PharmD, PhD

Connie A. Valdez, PharmD, MSEd, BCPS

Sumit Verma, MS, BPharm

Ami Vyas, PhD, MS, MBA

Rolin L. Wade, RPh, MS

David Wamble, PhD

Weijia Wang, MS

Yan Wang, PhD

Eric D. Weinhandl, PhD, MS

Arin Whitman, PharmD, BCOP
Peter Whittaker, PhD

Daniel Wiederkehr, MPH

Ryan Wiest, PharmD, MBA

Aaron Winn, PhD

Karen Worley, $\mathrm{PhD}$

Chung-Hsuen Wu, PhD

Joanne $\mathrm{Wu}, \mathrm{MD}, \mathrm{MS}$

Jun $\mathrm{Wu}, \mathrm{PhD}$

Tzu-Hua Wu, PhD

Jing Xie, MD, MPH, ScD

Yihua $\mathrm{Xu}, \mathrm{PhD}$

Hongbo Yang, PhD

Winnie M. Yang, PharmD

Xiaoqin Yang, $\mathrm{PhD}$

Paul Yoon, RPh

Peinie Young, PharmD

Jeffrey C. Yu, AB, MHS

Stephanie Yu, PharmD

Xueting Yu, PhD

Jing Yuan, BPharm

Autumn D. Zuckerman, PharmD 